\title{
Project Zero Obesity
}

\author{
A Rito ${ }^{1,2}$, MA Carvalho' ${ }^{2}$, C Ramos ${ }^{2}$ and J Breda ${ }^{2}$ \\ ${ }^{1}$ National Institute of Health Doutor Ricardo Jorge, Lisbon, Portugal: ${ }^{2}$ University Atlantica, Oeiras, Portugal
}

Introduction: Childhood obesity represents one of the most serious public health challenges, as it reached epidemic levels in several countries around the world. The prevention and treatment of this disease should be a top priority. The interventions used within childhood obesity approach should be community and family based and should essentially lie upon behaviour modification regarding diet and physical activity. The main purpose of the Project Zero Obesity is to tackle childhood obesity at municipality level through a set of activities (Healthy cooking programme and a nutritional guidance programme) targeted at lowincome families with overweight children (6-10 years old).

Method: A quasi-experimental multicentric study, developed in 2009/2010 in five Portuguese municipalities from the five regions of Portugal: Melgaco - north, Mealhada centre, Cascais - great Lisbon, Beja - alentejo articulated with healthcare centres and local governments. The program offered, to children and their families, a four stages intervention: (i) four sessions of Individual Nutrition Counselling, (ii) a Healthy Cooking workshop, (iii) two children's group sessions (nutrition and physical activity) and (iv) a Parents/ families Group counselling. Outcomes of nutritional status were assessed at baseline and at 6 months after.

Results: Of the 294 children participants in the intervention $(47.5 \%$ boys and $52.9 \%$ girls; mean age 8.6 years; mean percentile 93.6), 220 (75\%) have completed the programme. Mean percentile decreased by $2369(P<0 \cdot 05)$.

Conclusions: These data suggest that interventions at local level can have significant effects on childhood overweight prevalence. This knowledge may identify additional potential effective interventions in order to reverse the obesity trends in Portuguese children, one of the highest in Europe.

\section{A nutrition intervention program in preschool children in northern Greece}

\author{
M Hassapidou ${ }^{1,2}$, E Daskalou' ${ }^{1}$ A Paschaleri ${ }^{1}$, S Papadopoulou' ${ }^{1}$, Pagkalos ${ }^{1}$, \\ D Kaklamanou ${ }^{2}$ and Th Tzotzas ${ }^{1,2}$
}

${ }^{1}$ Nutrition and Dietetics Department, Alexander Technological Educational Institute, Thessaloniki, Greece: ${ }^{2} \mathrm{Hellenic}$ Medical Association for the Study of Obesity (HMSO)

Introduction: The rapid increase of childhood obesity all over Europe, and the impact in later life complications, creates the need of planning effective interventional nutritional programs from an early age. Developing programs to influence children's opportunities for healthy choices must be an important concern for nutrition scientists. The aim of the present study was to assess the effectiveness of a nutrition intervention program using educational methods, in preschool children, with emphasis on snack consumption.

Method: Fifty-four preschool children (twenty-seven boys and twenty-seven girls) participated in the study, aged 2-5 years (mean age 3.99 (SD 0.79) years) from Thessaloniki Municipality kindergartens, in northern Greece. Subjects were assigned into two equal numbered groups, intervention (IG) and control (CG) group. Eight educational courses concerning nutrition and healthy food choices were applied at the IG. The intervention lasted for 3 months. Furthermore, informative brochures with principles on normal growth, nutrition and food quality were provided for the parents of the IG children. Additionally the parents of all participants completed a detailed questionnaire at baseline, which comprised of questions regarding sociodemographic status, nutritional habits, behaviours and practices with possible effect on nutritional status of the children. Weight and height were measured and BMI was calculated, while weight categorization was performed using International Obesity Task Force (IOTF)'s criteria. Snack consumption was assessed using an FFQ. The same methodology including anthropometry and FFQ was applied at the beginning and after the completion of the intervention program. 